\begin{tabular}{|c|c|}
\hline Title & The mean pressure and density in a strongly nonlinear plane acoustic wave \\
\hline Author(s) & Y ano, Takeru \\
\hline Citation & $\begin{array}{l}\text { The Journal of the A coustical Society of A merica, 100(1), 666-668 } \\
\text { https://doi.org/10.1121/1.415964 }\end{array}$ \\
\hline Issue Date & 1996-07 \\
\hline Doc URL & http:/hdl.handle.net/2115/4907 \\
\hline Rights & $\begin{array}{l}\text { Copyright 1996, A coustical Society of A merica. This article may be downloaded for personal use only. A ny other use } \\
\text { requires prior permission of the author and the A coustical Society of A merica. }\end{array}$ \\
\hline Type & article \\
\hline File Information & JA SA 100-1.pdf \\
\hline
\end{tabular}

Instructions for use 


\title{
The mean pressure and density in a strongly nonlinear plane acoustic wave
}

\author{
Takeru Yano \\ Department of Engineering Science, Faculty of Engineering, Hokkaido University, Sapporo 060, Japan
}

(Received 17 November 1995; accepted for publication 2 April 1996)

\begin{abstract}
Exact expressions are presented for a time-averaged pressure and density at a fixed point in a strongly nonlinear plane acoustic wave radiated into an inviscid ideal gas of semi-infinite extent by a harmonic oscillation of an infinite plate. By making use of the exact solution of simple wave, the time averages of pressure and density are obtained up to the time of shock formation, in complete forms involving the hypergeometric functions. (C) 1996 Acoustical Society of America.
\end{abstract}

PACS numbers: 43.25.Qp [MAB]

\section{INTRODUCTION}

Time averages of pressure and density at a fixed point in the nonlinear plane progressive waves were examined by the leading pioneers of nonlinear acoustics. ${ }^{1-5}$ They expanded the time averages in infinite series in wave amplitude, and evaluated first several terms in the series. Such truncated formulas can only be applied to the case that the nonlinearity is sufficiently weak, i.e., the acoustic Mach number $M$ defined as

$$
M=u_{0} / c_{0},
$$

is sufficiently small compared with unity, where $u_{0}$ is the maximum speed at the sound source and $c_{0}$ is the speed of sound in an undisturbed fluid.

Recently, the strongly nonlinear propagation process characterized by the conditions

$$
M=O(1) \text { and } \operatorname{Re} \gg 1,
$$

has been analyzed in Ref. 6 ( $\operatorname{Re}$ is the acoustic Reynolds number ${ }^{7}$ ), and as a result, several strongly nonlinear phenomena have been found, which present striking contrasts to the results of the conventional weakly nonlinear theory. Among the strongly nonlinear phenomena, acoustic streaming (mean mass flow) due to the presence of shocks and resulting decrease in the density of the gas near the source may be deserving special attention, because whether the wave motion can reach a steady state or not may strongly be affected by them; this will be discussed in detail in a forthcoming paper.

Clearly, the truncated formulas of mean values obtained by the pioneers are insufficient for the analysis of the strongly nonlinear problem. In the following, we shall evaluate the mean pressure and mean density at a fixed point in complete forms.

\section{EXACT SOLUTION OF SIMPLE WAVE}

We shall consider the propagation of nonlinear plane acoustic waves in an inviscid ideal gas, up to the time of shock formation. The gas is supposed to be initially uniform and at rest. At the time $t^{*}=0$, the plate starts executing the harmonic oscillation of amplitude $a$ and angular frequency $\omega$, and thus the plane acoustic wave is emitted into the gas of semi-infinite extent (see Fig. 1).

We shall introduce the nondimensional variables

$$
t=\omega t^{*}, \quad x=\frac{x^{*} \omega}{c_{0}}, \quad u=\frac{u^{*}}{c_{0}}, \quad \rho=\frac{\rho^{*}}{\rho_{0}}, \quad p=\frac{p^{*}}{\gamma p_{0}},
$$

where $x^{*}$ is the distance from an initial position of the plate, $u^{*}$ is the $x^{*}$ component of the fluid velocity, $\rho^{*}$ is the density of the gas, and $p^{*}$ is the pressure $\left(\rho_{0}\right.$ and $p_{0}$ are the initial undisturbed density and pressure, and $\gamma$ is the ratio of specific heats of ideal gas).

Since the gas is uniform and at rest for $t \leqslant 0$, the initial conditions at $t=0$ are

$$
u=0, \quad \rho=1, \quad p=1 / \gamma \quad(x \geqslant 0) .
$$

The boundary conditions on the plate is given by

$$
u=-M \sin t \quad \text { at } \quad x=M(\cos t-1) \quad \text { for } t \geqslant 0,
$$

where $M=a \omega / c_{0}$ is the acoustic Mach number. The weakly nonlinear waves and the strongly nonlinear waves are characterized by the conditions $M \ll 1$ and $M=O(1)$, respectively.

Up to the shock formation time, the wave is the simple wave, ${ }^{8}$ and the exact solution of simple wave satisfying the mass and momentum conservation laws, the isentropic relation, initial condition (4), and boundary condition (5) can be obtained by the method of characteristics: ${ }^{4,6,9}$

$$
\begin{aligned}
& u=-M \sin \mu, \\
& t-x=\mu-M(\cos \mu-1)+\frac{\gamma+1}{2} M(t-\mu) \sin \mu,
\end{aligned}
$$

where $\mu$ is a parameter indicating the time when a characteristic in the $(x, t)$ plane [the second of Eq. (6)] is issued from the plate. Once the function $u(x, t)$ has been found from Eq. (6), we can readily have other quantities as functions of $x$ and $t$ :

$p=\frac{1}{\gamma}\left(1+\frac{\gamma-1}{2} u\right)^{2 \gamma /(\gamma-1)}, \quad \rho=\left(1+\frac{\gamma-1}{2} u\right)^{2 /(\gamma-1)}$. 


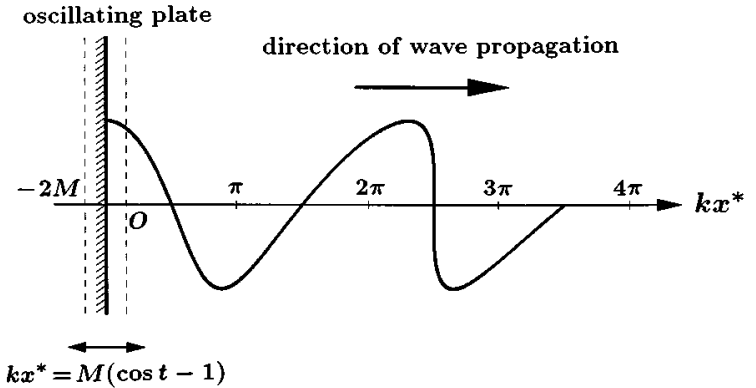

FIG. 1. Schematic of the model: generation of nonlinear plane acoustic wave by an oscillating infinite plate.

Here, the first of Eq. (7) is the isentropic relation for the ideal gas and the second is derived from the fact that a Riemann invariant $u-2 c /(\gamma-1)$ is equal to $-2 /(\gamma-1)$ everywhere ( $c=\sqrt{\gamma p / \rho}$ is the nondimensional local speed of sound).

The velocity profile described by Eq. (6) is distorted as the wave propagates, and this waveform distortion eventually leads to the formation of shock wave at the smallest time $t_{s}$ satisfying $\partial u / \partial x=-\infty$. The time $t_{s}$ and distance $x_{s}$ of shock formation have been analyzed in Ref. 6, by using Eq. (6).

\section{MEAN PRESSURE AND DENSITY}

By making use of the exact solution of simple wave presented in Sec. I, we shall evaluate the time-averaged pressure and density defined as

$$
\bar{p}=\frac{1}{2 \pi} \int_{t-2 \pi}^{t} p(x, \tau) d \tau
$$

and

$$
\bar{\rho}=\frac{1}{2 \pi} \int_{t-2 \pi}^{t} \rho(x, \tau) d \tau,
$$

for $0 \leqslant x \leqslant t-2 \pi$ and $t \leqslant t_{s}$. To simplify the final results, we introduce a parameter $N$,

$$
N=\frac{\gamma+1}{2 \gamma-2}, \quad \text { i.e., } \quad \gamma=\frac{2 N+1}{2 N-1}
$$

( $N$ is not necessarily an integer). Since we take the ideal gas, $1<\gamma \leqslant 5 / 3$, and hence $N \geqslant 2$. The air $\gamma=1.4$ corresponds to the case of $N=3$. Substituting Eq. (9) into the second of Eq. (6) and differentiating it with respect to $\mu$ with $x$ being fixed, we have

$$
\frac{d t}{d \mu}=1+\frac{[2 N /(2 N-1)] M(M+x) \cos \mu+M \sin \mu-[2 N /(2 N-1)] M^{2}}{(1-[2 N /(2 N-1)] M \sin \mu)^{2}} .
$$

Substituting Eq. (7) into the definitions of $\bar{p}$ and $\bar{\rho}$, we can rewrite Eq. (8) into

$$
\bar{p}=\frac{1}{2 \pi} \int_{0}^{2 \pi} \frac{2 N-1}{2 N+1}\left(1+\frac{M}{2 N-1} \sin \mu\right)^{2 N+1} \frac{d t}{d \mu} d \mu,
$$

and

$$
\bar{\rho}=\frac{1}{2 \pi} \int_{0}^{2 \pi}\left(1+\frac{M}{2 N-1} \sin \mu\right)^{2 N-1} \frac{d t}{d \mu} d \mu
$$

where the range of integration is shifted to $[0,2 \pi]$, since the integrands are composed of $\sin \mu$ and $\cos \mu$ [cf. Eq. (10)].

Substituting Eq. (10) into Eqs. (11) and (12), expanding the integrands into the power series of $M$, and integrating the resultant equations term by term, we obtain

$$
\begin{aligned}
\bar{p}= & \frac{2 N-1}{2 N+1} F\left(-\frac{1}{2}-N,-N, 1 ; \frac{M^{2}}{(2 N-1)^{2}}\right) \\
& -\frac{1}{2} M^{2} F\left(\frac{1}{2}-N,-N, 2 ; \frac{M^{2}}{(2 N-1)^{2}}\right) \\
& +\left(\frac{2 N-1}{2 N}\right)^{2(N+1)}\left[\sqrt{1-\left(\frac{2 N M}{2 N-1}\right)^{2}}-1+\frac{1}{2}\left(\frac{2 N M}{2 N-1}\right)^{2}\right]
\end{aligned}
$$

$$
\begin{aligned}
& +\frac{M^{2}}{2} \sum_{n=1}^{\infty} \frac{\Gamma\left(n+\frac{1}{2}-N\right) \Gamma(n-N)}{\Gamma\left(\frac{1}{2}-N\right) \Gamma(-N) n !(n+1) !}\left(\frac{M}{2 N-1}\right)^{2 n} \\
& \times F\left(2 n-2 N, 1,2 n+1 ; \frac{1}{2 N}\right),
\end{aligned}
$$

$$
\begin{aligned}
\bar{\rho}= & F\left(\frac{1}{2}-N, 1-N, 1 ; \frac{M^{2}}{(2 N-1)^{2}}\right) \\
& -\frac{1}{2} M^{2} F\left(\frac{3}{2}-N, 1-N, 2 ; \frac{M^{2}}{(2 N-1)^{2}}\right) \\
& +\left(\frac{2 N-1}{2 N}\right)^{2 N}\left[\sqrt{1-\left(\frac{2 N M}{2 N-1}\right)^{2}}-1+\frac{1}{2}\left(\frac{2 N M}{2 N-1}\right)^{2}\right] \\
& +\frac{M^{2}}{2} \sum_{n=1}^{\infty} \frac{\Gamma\left(n+\frac{3}{2}-N\right) \Gamma(n+1-N)}{\Gamma\left(\frac{3}{2}-N\right) \Gamma(1-N) n !(n+1) !}\left(\frac{M}{2 N-1}\right)^{2 n} \\
& \times F\left(2 n+2-2 N, 1,2 n+1 ; \frac{1}{2 N}\right),
\end{aligned}
$$

where $\Gamma$ is the gamma function and $F(a, b, c ; z)$ is the hypergeometric function. In calculating Eqs. (13) and (14), Wallis's formula has been used. Note that the term by term integration is allowed within the convergence radius of power series, i.e., $M<(2 N-1) / 2 N,(M<5 / 6$ for $\gamma=1.4)$. On the 
other hand, the use of the simple-wave solution requires that $t_{s}>2 \pi$; this imposes the severer restriction for $M$ than the convergence radius ( $M$ should therefore be less than 0.25 for $\gamma=1.4$, see Ref. 6). Accordingly, the results, Eqs. (13) and (14), are valid for $0 \leqslant x \leqslant t-2 \pi$ and $t \leqslant t_{s}$ in the case of $t_{s}>2 \pi$. We shall remark that $\bar{p}$ and $\bar{\rho}$ are independent of not only $t$ but also $x$.

For the case of air, $N=3$, Eqs. (13) and (14) can be rewritten into

$$
\begin{aligned}
\bar{p}= & \frac{5}{7}-\frac{1}{5} M^{2}+\frac{73}{259200} M^{4}+\frac{1}{360000} M^{6} \\
& +\left(\frac{5}{8}\right)^{8}\left[\sqrt{1-\left(\frac{6 M}{5}\right)^{2}}-1+\frac{1}{2}\left(\frac{6 M}{5}\right)^{2}\right] \\
& +\frac{M^{2}}{2} \sum_{n=4}^{\infty} \frac{\Gamma\left(n-\frac{5}{2}\right) \Gamma(n-3)}{\Gamma\left(-\frac{5}{2}\right) \Gamma(-3) n !(n+1) !}\left(\frac{M}{5}\right)^{2 n} \\
& \times F\left(2 n-6,1,2 n+1 ; \frac{1}{6}\right), \\
\bar{\rho}= & 1-\frac{3}{10} M^{2}-\frac{1}{18000} M^{4}+\left(\frac{5}{6}\right)^{6} \\
& \times\left[\sqrt{1-\left(\frac{6 M}{5}\right)^{2}}-1+\frac{1}{2}\left(\frac{6 M}{5}\right)^{2}\right] \\
& +\frac{M^{2}}{2} \sum_{n=3}^{\infty} \frac{\Gamma\left(n-\frac{3}{2}\right) \Gamma(n-2)}{\Gamma\left(-\frac{3}{2}\right) \Gamma(-2) n !(n+1) !}\left(\frac{M}{5}\right)^{2 n} \\
& \times F\left(2 n-4,1,2 n+1 ; \frac{1}{6}\right) .
\end{aligned}
$$

In the case that the nonlinearity is weak, i.e., $M \ll 1$, we can truncate the infinite series and obtain

$$
\bar{p}=\frac{2 N-1}{2 N+1}+\frac{1-N}{4 N-2} M^{2}+O\left(M^{4}\right)
$$

and

$$
\bar{\rho}=1+\frac{N}{2-4 N} M^{2}+O\left(M^{4}\right) .
$$

For the case of air, $N=3$, we have

$$
\bar{p}=\frac{5}{7}-\frac{1}{5} M^{2}+O\left(M^{4}\right) \quad \text { and } \quad \bar{\rho}=1-\frac{3}{10} M^{2}+O\left(M^{4}\right) .
$$

These are coincident with the results by the pioneers.

\section{CONCLUDING REMARKS}

We have presented the exact formulas for the mean pressure and density. Here we would like to give some relevant remarks: way: ${ }^{6}$

(i) Exact mean velocity can also be obtained in a similar

$$
\begin{aligned}
\bar{u} & =\frac{1}{2 \pi} \int_{t-2 \pi}^{t} u(x, \tau) d \tau \\
& =\left(\frac{2 N-1}{2 N}\right)^{2}\left[\sqrt{1-\left(\frac{2 N M}{2 N-1}\right)^{2}}-1\right],
\end{aligned}
$$

which has already been derived by Blackstock. ${ }^{4}$

(ii) The mean energy flux $\mathscr{E}$ and the mean momentum flux $\mathscr{A b}$ can also be obtained, in a similar way, as ${ }^{10}$

$$
\begin{aligned}
\mathscr{E} & =\frac{1}{2 \pi} \int_{t-2 \pi}^{t}\left(E_{t}+p\right) u d t \\
& =\frac{1}{2} M^{2} F\left(\frac{1}{2}-N,-N, 2 ; \frac{M^{2}}{(2 N-1)^{2}}\right),
\end{aligned}
$$

where $E_{t}=(1 / 2) \rho u^{2}+p /(\gamma-1)$ is the nondimensional total energy of the ideal gas per unit volume, and

$$
\begin{aligned}
\mathscr{C l} & =\frac{1}{2 \pi} \int_{t-2 \pi}^{t}\left(\rho u^{2}+p-\frac{1}{\gamma}\right) d t \\
& =\frac{2 N-1}{2 N+1}\left[F\left(-\frac{1}{2}-N,-N, 1 ; \frac{M^{2}}{(2 N-1)^{2}}\right)-1\right] .
\end{aligned}
$$

These are exactly reduced to polynomials in the case of air, $N=3$, as follows:

$$
\begin{aligned}
& \mathscr{E}=\frac{1}{2} M^{2}+\frac{3}{40} M^{4}+\frac{3}{2000} M^{6}+\frac{1}{4 \times 10^{5}} M^{8}, \\
& \mathscr{L}=\frac{3}{10} M^{2}+\frac{3}{200} M^{4}+\frac{1}{10000} M^{6} .
\end{aligned}
$$

In the weakly nonlinear limit, we have $\mathscr{E}=(1 / 2) M^{2}$, which is equal to the formula for the acoustic intensity.

(iii) $\bar{\rho}$ is less than its initial undisturbed value 1 . This does not mean that the gas is rarefied, because, up to the time of shock formation, the mean mass flow $\overline{\rho u}$ is exactly zero. ${ }^{6}$ The result, $\bar{\rho}<1$, is merely the reflection of the fact that the plate recedes from $x=0$ to $-2 M$ during a period $2 \pi$.

\section{ACKNOWLEDGMENT}

The author wishes to thank Professor Yoshinori Inoue for his continual encouragement.

${ }^{1} \mathrm{P}$. J. Westervelt, "The mean pressure and velocity in a plane acoustic wave in a gas," J. Acoust. Soc. Am. 22, 319-327 (1950).

2 J. J. Markham, "'Second-order acoustic fields: relations between energy and intensity,', Phys. Rev. 89, 972-977 (1953).

${ }^{3} \mathrm{~F}$. V. Hunt, "Notes on the exact equations governing the propagation of sound in fluids,' J. Acoust. Soc. Am. 27, 1019-1039 (1955).

${ }^{4}$ D. T. Blackstock, "Propagation of plane sound waves of finite amplitude in nondissipative fluids,' J. Acoust. Soc. Am. 34, 9-30 (1962).

${ }^{5}$ L. D. Landau and E. M. Lifshitz, Fluid Mechanics Second Edition (Pergamon, Oxford, 1987), Sec. 101, problem 4, pp. 384-385.

${ }^{6}$ Y. Inoue and T. Yano, "Propagation of strongly nonlinear plane waves," J. Acoust. Soc. Am. 94, 1632-1642 (1993); "Propagation of acoustic shock waves of large amplitude,' in Frontiers of Nonlinear Acoustics, edited by M. F. Hamilton and D. T. Blackstock (Elsevier, London, 1990), pp. $141-146$.

${ }^{7}$ O. V. Rudenko and S. I. Soluyan, Theoretical Foundations of Nonlinear Acoustics (Consultant Bureau, New York, 1977).

${ }^{8}$ R. Courant and K. O. Friedrichs, Supersonic Flow and Shock Waves (Springer-Verlag, New York, 1985), Sec. 40.

${ }^{9} \mathrm{~S}$. Earnshaw, "On the mathematical theory of sound,' Trans. R. Soc. London 150, 133-148 (1860).

${ }^{10} \mathrm{Y}$. Inoue and T. Yano, "Propagation of strongly nonlinear plane $N$ waves", J. Fluid Mech. (submitted). 